\title{
Prognostic significance of blood coagulation tests in lung cancer
}

\author{
D. Ferrigno*, G. Buccheri*, I. Ricca\#
}

Prognostic significance of blood coagulation tests in lung cancer. D. Ferrigno, G. Buccheri, I. Ricca. (C) ERS Journals Ltd 2001.

ABSTRACT: Previous studies have shown that activation of coagulation has an impact on the clinical course of lung cancer.

This study was carried out to assess the potential prognostic significance of platelet count $(\mathrm{P})$, prothrombin time (PT), partial thromboplastin time (PTT), anti-thrombin III (AT-III), fibrinogen (F), D-dimer (DD), factor II (F-II), factor VII (F-VII), factor X (F$\mathrm{X}$ ), protein C clotting (PCC), plasminogen (PL), and antiplasmin (AP) in 343 consecutive new lung cancer patients. A set of 32 anthropometric, clinical, physical, laboratory, radiological, and pathological variables was recorded prospectively for all patients. Patients were carefully followed-up, and their subsequent clinical course recorded.

The most frequent abnormalities were of DD, F, and AT-III followed by F-VII, F-X, and F-II. Among the 12 clotting variables, the strongest relationships were those of F-II and F-X (Spearman rank $(\mathrm{rs})=0.565)$, PT and F-VII $(\mathrm{rs}=0.562)$, F-VII and F-X $(r s=0.514), P L$ and AP $(r s=0.515), F$ and $P(r s=0.490)$, AT-III and PCC $(r s=0.476)$. Univariate analyses of survival showed that prolonged PT $(\mathrm{p}<0.043)$, and abnormally elevated DD $(\mathbf{p}<0.003), F(p<0.031)$, and $P(p<0.047)$ were all associated with a poor prognosis. The multivariate model, however, did not confirm the prognostic significance of the coagulation factors.

The results show subclinical activation of blood coagulation in lung cancer patients with early disease. In addition, clotting activation is confirmed as a predictor of survival, although not independently of other prognostic factors.

Eur Respir J 2001; 17: 667-673.

*Division of Respiratory Medicine, $\mathrm{S}$ Croce e Carle Hospital, Cuneo. ${ }^{\#}$ Dept of Oncology-Haemathology, San Giovanni Battista Hospital, Torino, Italy.

Correspondence: D. Ferrigno

Divisione di Pneumologia

Ospedale "S. Croce e Carle"

I-12100 Cuneo

Italy

Fax: 39171441764

Keywords: Blood coagulation

lung cancer

prethrombotic state

prognosis

Received: May 92000

Accepted after revision November 8 2000

This study was funded by the Cuneo Lung Cancer Study Group

Following the first reports by Trousseau [1], biological data and several experiments studies have confirmed an important relationship between neoplasia and phenomena related to blood coagulation [2-4]. Generally, patients with cancer show abnormalities of one or more circulating markers of haemostasis activation, underlying a hypercoagulable state. However, the biochemical basis of this phenomenon is not completely understood $[4,5]$. The potential role of the clotting-fibrinolytic system in the pathogenesis of malignancy, tumour growth regulation and cancer cell dissemination has previously been suggested [2, 3]. Anticoagulant therapy has been reported to suppress the invasion of cancer cells in experimental models and to confer a survival benefit in some types of tumour when used in combination with chemotherapy [6-8]. Also in lung cancer, systemic activation of the clotting system frequently occurs [2-4]. Clotting abnormalities have been shown to correlate with tumour burden, clinical progression, and the response to chemotherapy $[2,4,7,9,10]$. It has recently been reported that a number of coagulation factors (e.g. prothrombin time, fibrinogen, and D-dimer) are important independent predictors of survival [3].

The purpose of the current study was to: a) confirm the previous data; b) assess other coagulation-fibrino- lytic factors and evaluate their sub clinical activation; c) define the possible correlation between such abnormalities and other clinical and laboratory characteristics; and d) investigate the prognostic value of pretreatment plasma levels of such haemostatic abnormalities.

\section{Patients and methods}

\section{Patients}

From January 1996 to December 1999, 343 consecutive unselected patients (304 males and 39 females, median (range) age 68 (39-86) with histologically proven bronchogenic carcinoma, were referred to the division of Respiratory Diseases of the "S. Croce and A. Carle Hospital" of Cuneo. The pathological diagnosis of primary lung cancer was made in accordance with the revised World Health Organization (WHO) classification of lung tumours [11] and included 125 squamous cell carcinomas, 80 adenocarcinomas, 34 small cell carcinomas, 16 large cell carcinomas, and 88 unclassified carcinomas. All patients were classified according to the 1997 staging system [12]. Pretreatment clinical evaluation was based on physical examination, weight loss determination and the Eastern Co-operative 
Oncology Group (ECOG) performance status [13]. A battery of laboratory tests, including coagulation tests, was routinely requested. Further investigations included radiography, fibreoptic bronchoscopy and computed tomography of chest, upper abdomen and brain. In potentially resectable tumours, any radiological finding equivocal for nodal mediastinal involvement was considered an indication for mediastinoscopy. Patients with dubious metastatic involvement were further investigated with appropriate imaging studies, biopsies or needle aspirations. Bone scan and bone marrow biopsy were performed in most small cell lung cancer (SCLC) patients. As expected, only a relatively small fraction of patients could be treated surgically (19\% of the cohort). Chemotherapy was the most frequent treatment, in accordance with the early recognition of its potential benefit $[14,15]$ either alone or in combination ( $51 \%$ of the population). Curative radiotherapy was attempted in only $2 \%$ of the cases; the remaining patients received the best supportive care $(26 \%)$. Survival was recorded from the time of histological diagnosis to death, or to the last clinical examination or telephone contact with the patient, the family, the family doctor, or the municipal registry office. Consequently, both the duration of survival, measured from the first hospital admission or outpatient examination, and the status of dead or alive at the closure of the study (i.e. at the end of December 1999) was recorded for all patients. At this point, 71 patients $(21 \%)$ were still alive, after a median follow-up time of 8.5 months (quartile range 3.6-17.4).

\section{Study design}

This was a prospective study. Thirty-two variables for each patient entered on study were recorded, including the following twelve coagulation factors: platelet count $(\mathrm{P})$, prothrombin time $(\mathrm{PT})$, partial thromboplastin time (PTT) and concentrations of antithrombin III (AT-III), fibrinogen (F), D-dimer (DD), factor II (F-II), factor VII (F-VII), factor X (F-X), protein $\mathrm{C}$ clotting (PCC), plasminogen (PL), and antiplasmin (AP). The other variables recorded were age, sex, ECOG performance status, weight loss, haemoglobin concentration, total white cell/neutrophil counts, tumour cell type, clinical stage, and the single tumour, node and metastasis factors, analysed individually for their prognostic meaning. In addition, the serum concentration of each of the following were measured and recorded: lactate dehydrogenase, alkaline phosphatase, pyruvic and oxalacetic transaminases, creatinine, sodium, carcinoembryonic antigen, and tissue polypeptide antigen [16]. Follow-up consisted of clinical, laboratory, and radiological reassessments at 3-4 week intervals during chemotherapy, and every 3-6 weeks in case of palliative radiotherapy, or no anticancer treatment. Patients treated with radical surgery were scheduled for examination at longer intervals of 3-6 months.

\section{Coagulation assays}

Commercially available reagents provided by the Diagnostica stago (Boehringer Mannheim Co., Ger- many) were used to measure PT (STA Neoplastin Plus kit), PTT (STA APTL LT kit), AT-III (STA Antithrombin III kit), F-II (STA Factor II kit), F-VII (STA Factor VII kit), F-X (STA Factor X kit), PCC (STA Protein C Clotting kit), DD (STA LIATEST D-DI kit), and F (STA Fibrinogen kit). Regents provided by Diagnostica stago (France) were used to measure plasminogen (STA-STACHROM PLASMINOGEN kit) and antiplasmin (STA-STACHROM ANTIPLASMIN kit). For all determinations, the manufacturer's instructions were followed. Patients' blood samples were obtained at presentation and processed immediately, or stored for a maximum of 2 days at $-20^{\circ} \mathrm{C}$ until needed.

\section{Statistical analysis}

Statistical analysis was performed using the Statistical Package for Social Sciences (SPSS) package for Windows, Version 9.0 (SPSS Inc., Chicago, IL, USA). Nonparametric tests [17] were used to assess relationships and differences among coagulation factors or between these factors and the other variables recorded (i.e. Spearman rank, Kruskall-Wallis, and median test, as appropriate). Survival curves were constructed using the Kaplan-Meier method [18] and compared by means of the log-rank test [19]. Multivariate survival analyses were performed using the Cox's proportional hazards regression model [20]. Continuous variables were categorized using the median value as cut-point. The significance of each factor was calculated by the maximum likelihood ratio. A p-value of 0.1 was set to enter, while a value of 0.15 was set to remove a variable from the model. A p-value of $<0.05$ was regarded as statistically significant. All tests were two-sided.

\section{Results}

Almost all the pretreatment laboratory parameters for this study plus nine clinical variables and the twelve coagulation tests were available for the 343 documented patients with lung cancer. The number of observations and, either the frequency or the median and range for all the clinical and laboratory variables are shown in table 1. Similar information plus the number of normal, low, and abnormally high values for all clotting tests are given in table 2 .

\section{Coagulation factors and correlation with other clinical and laboratory variables}

Forty-four patients $(13 \%)$ had manifest thrombocytosis $\left(=>400,000 \cdot \mathrm{mm}^{-3}\right)$, while nine $(3 \%)$ were thrombocytopenic $\left(<150,000 \cdot \mathrm{mm}^{-3}\right)$. In 15 cases $(4 \%)$ PT was $<70 \%$ of the reference value. Ten patients $(3 \%)$ had frankly reduced PTT $(<25 \mathrm{~s})$, and in only seven was PTT $(2 \%)$ lower than $40 \mathrm{~s}$. F-II, F-VII, F-X were abnormal $(>100 \%)$ in $55(16 \%), 90(27 \%)$, and $66(20 \%)$ patients, respectively. Increased values of F, DD, and AT-III were found in $143(42 \%), 182(55 \%)$, and 92 
Table 1. - Anthropometric, clinical and haematological characteristics of the study population

\begin{tabular}{|c|c|c|}
\hline Characteristic & Observations & Median (range) or Frequency \\
\hline Age yrs & 343 & $68(39-86)$ \\
\hline $\operatorname{Sex} \mathrm{M} / \mathrm{F}$ & 343 & $304 / 31$ \\
\hline ECOG PS 0/1/2/3/4 & 343 & 43/157/108/28/7 \\
\hline Weight loss in the prior 6 months $\mathrm{Y} / \mathrm{N}$ & 343 & 244/99 \\
\hline Haemoglobin $\mathrm{g} \cdot \mathrm{dL}^{-1}$ & 343 & $13.5(8.0-20.0)$ \\
\hline White blood cells $\mathrm{n} \cdot \mathrm{mm}^{-3}$ & 343 & $9416(3300-30700)$ \\
\hline Neutrophils $\mathrm{n} \cdot \mathrm{mm}^{-3}$ & 343 & $6622(1600-24100)$ \\
\hline Lactate dehydrogenase $\mathrm{mg} \cdot \mathrm{dL}^{-1}$ & 343 & $432(81-4895)$ \\
\hline Alkaline phosphatase $\mathrm{mg} \cdot \mathrm{dL}^{-1}$ & 341 & $114(43-2171)$ \\
\hline Glutamic pyruvic transaminase $\mathrm{mg} \cdot \mathrm{dL}^{-1}$ & 343 & $28(5-202)$ \\
\hline Glutamic oxalacetic transaminase $\mathrm{mg} \cdot \mathrm{dL}^{-1}$ & 343 & $26(9-208)$ \\
\hline Creatinine $\mathrm{mg} \cdot \mathrm{dL}^{-1}$ & 343 & $1(0.5-3.3)$ \\
\hline Sodium $\mathrm{mEq} \cdot \mathrm{mL}^{-1}$ & 342 & $140(114-148)$ \\
\hline Carcinoembryonic antigen $\mathrm{ng} \cdot \mathrm{mL}^{-1}$ & 342 & $35(0-1854)$ \\
\hline Tissue polypeptide antigen $\mathrm{U} \cdot \mathrm{L}^{-1}$ & 342 & $203(30-2900)$ \\
\hline Histology E/S/A/L/U & 343 & $125 / 34 / 80 / 16 / 88$ \\
\hline Stage of disease $0 / 1 a / 1 b / 2 a / 2 b / 3 a / 3 b / 4$ & 343 & $3 / 35 / 34 / 5 / 21 / 40 / 84 / 121$ \\
\hline $\mathrm{T}$ factor $0 / 1 / 2 / 3 / 4$ & 343 & 3/63/118/51/108 \\
\hline $\mathrm{N}$ factor $0 / 1 / 2 / 3$ & 343 & $151 / 38 / 107 / 47$ \\
\hline M factor $0 / 1$ & 343 & $222 / 121$ \\
\hline
\end{tabular}

ECOG: Eastern Cooperative Oncology Group; PS: performance status; Y: yes; N: no; E: epidermoid carcinomas; S: small cell lung cancers; A: adenocarcinomas; L: large cell anaplastic carcinomas; U: unclassified carcinomas; T factor: single tumour factor; $\mathrm{N}$ factor: node factor; $\mathrm{M}$ factor: metastasis factor.

(28\%) cases, respectively. A small number of patients had increased values of PL $(8 \%)$, PCC $(6 \%)$, and AP $(3 \%)$. No coagulation factor showed abnormalities related to the cell type ( $\mathrm{p}$-values for the differences varied $0.49-0.07$, Kruskall-Wallis test). The strongest relationships, between clinical and laboratory variables were those of ECOG-PS with weight-loss (Spearman $r$ $(r s)=0.236)$, and stage of disease $(r s=0.109)$. Other significant associations were seen between ECOG PS and AT-III ( $\left.\mathrm{rs}_{\mathrm{s}}=-0.157\right), \mathrm{F}(\mathrm{rs}=0.142), \mathrm{F}-\mathrm{II}(\mathrm{rs}=0.141), \mathrm{F}$ $\mathrm{X}\left(\mathrm{rs}_{\mathrm{s}}=0.136\right)$, and DD $\left(\mathrm{rs}_{\mathrm{s}}=0.113\right)$. The stage of disease was related to $\mathrm{P}\left(\mathrm{rs}_{\mathrm{s}}=0.124\right)$ and $\mathrm{F}\left(\mathrm{rs}_{\mathrm{s}}=0.130\right)$, while weight loss was correlated with $\mathrm{F}\left(\mathrm{r}_{\mathrm{s}}=0.131\right)$ and AT-III $(\mathrm{rs}=0.120)$. Bivariate correlation tests among the 12 clotting variables revealed several significant associations (table 3). In particular, the strongest relationships were those between F-II and F-X ( $\left.\mathrm{rs}_{\mathrm{s}}=0.565\right)$, PT and FVII ( $\left.\mathrm{r}_{\mathrm{s}}=0.562\right), \mathrm{F}$-VII and F-X ( $\left.\mathrm{rs}=0.514\right), \mathrm{PL}$ and AP $\left(\mathrm{r}_{\mathrm{s}}=0.515\right), \mathrm{F}$ and $\mathrm{P}\left(\mathrm{rs}_{\mathrm{s}}=0.490\right), \mathrm{AT}-\mathrm{III}$ and PCC $\left(r_{s}=0.476\right)$. Other frequent and significant relationships were those between F-II, F-VII, F-X and PCC, PL, AP,
AT-III (table 3). DD was related only to F-II and AP $(\mathrm{rs}=-0.113 /-0.112)$.

\section{Survival analysis}

Univariate analyses of survival confirmed the already known relationships between survival and stage of disease, TNM factors, ECOG PS and weight loss (pvalue for each $<0.001)$. Conversely, AP $(p=0.987)$, PL $(p=0.935), \quad$ F-II $(p=0.465), \quad$ PTT $(p=0.458), \quad$ PCC $(p=0.297), F-X(p=0.173)$, AT-III $(p=0.115)$, and FVII $(p=0.114)$ were unrelated to the patients' outcome. Patients with values of fibrinogen $<447 \mathrm{mg} \cdot \mathrm{dL}^{-1}$ had significantly longer survival than those with values above the median (10 months, $95 \%$ confidence intervals (CI): 7-13, versus 8 months, CI: 7-10, p<0.031) (fig. 1a). Similarly, abnormal elevated concentrations of DD were strongly predictive of a poor prognosis (7 versus 10 months, CI: $6-9$ and $8-12$ respectively, $\mathrm{p}<0.003$ ) (fig. 1b). Patients with PT values $>90 \%$ had a shorter

Table 2. - Coagulation variables

\begin{tabular}{|c|c|c|c|c|}
\hline Variable & Reference range & Observations & $\begin{array}{l}\text { Median (range) } \\
\text { and frequency }\end{array}$ & $\begin{array}{c}\text { Observations } \\
\text { normal/low/high }\end{array}$ \\
\hline Platelets & $150-400 \cdot \mathrm{mm}^{-3} \times 1000$ & 343 & $289(93-768)$ & $290 / 9 / 44$ \\
\hline Prothrombin time & $70-100 \%$ & 342 & $90(16-100)$ & $327 / 15$ \\
\hline Partial thromboplastin time & $25-30 \mathrm{~s}$ & 342 & $31(23-52)$ & $325 / 10 / 7$ \\
\hline Antithrombin III & $70-100 \%$ & 331 & $93(43-124)$ & $226 / 13 / 92$ \\
\hline Protein $\mathrm{C}$ clotting & $60-140 \%$ & 333 & $106(35-200)$ & $309 / 3 / 21$ \\
\hline Fibrinogen & $150-450 \mathrm{mg} \cdot \mathrm{dL}^{-1}$ & 337 & $447(126-892)$ & $193 / 1 / 143$ \\
\hline D-dimer & $<0.5 \mu \mathrm{g} \cdot \mathrm{mL}^{-1}$ & 333 & $0.9(0.1-4)$ & $151 / 182$ \\
\hline Factor II & $70-100 \%$ & 337 & $94(24-127)$ & $274 / 8 / 55$ \\
\hline Factor VII & $70-100 \%$ & 338 & $92(22-163)$ & $217 / 31 / 90$ \\
\hline Factor X & $70-100 \%$ & 338 & $94(49-134)$ & $256 / 16 / 66$ \\
\hline Plasminogen & $75-125 \%$ & 325 & $103(58-148)$ & $289 / 75 / 27$ \\
\hline Antiplasmin & $80-120 \%$ & 327 & $100(60-140)$ & $309 / 8 / 10$ \\
\hline
\end{tabular}


Table 3. - Correlation tests among coagulation factors (Spearman $\rho$ )

\begin{tabular}{lcccccccccccc}
\hline & PT & PTT & F & F-II & F-VII & F-X & DD & PCC & PL & AP & AT-III & P \\
\hline PT & 1.000 & $-0.242^{*}$ & -0.077 & $0.220^{*}$ & $0.562^{*}$ & $0.368^{*}$ & -0.061 & $0.310^{*}$ & $0.132^{*}$ & 0.060 & $0.179^{*}$ & $-0.107^{*}$ \\
PTT & $-0.242^{*}$ & 1.000 & $0.113^{*}$ & $-0.188^{*}$ & $-0.192^{*}$ & $-0.239^{*}$ & -0.052 & $-0.224^{*}$ & -0.011 & $-0.207^{*}$ & $-0.139^{*}$ & 0.095 \\
F & -0.077 & $0.113^{*}$ & 1.000 & $0.128^{*}$ & -0.062 & -0.005 & -0.032 & -0.011 & $0.328^{*}$ & $0.108^{*}$ & $-0.121^{*}$ & $0.490^{*}$ \\
F-II & $0.22^{*}$ & $-0.188^{*}$ & $0.128^{*}$ & 1.000 & $0.319^{*}$ & $0.565^{*}$ & $-0.113^{*}$ & $0.349^{*}$ & $0.284^{*}$ & $0.310^{*}$ & $0.323^{*}$ & $0.179^{*}$ \\
F-VII & $0.562^{*}$ & $-0.192^{*}$ & -0.062 & $0.319^{*}$ & 1.000 & $0.514^{*}$ & -0.10 & $0.399^{*}$ & $0.232^{*}$ & $0.186^{*}$ & $0.272^{*}$ & $-0.127^{*}$ \\
F-X & $0.368^{*}$ & $-0.239^{*}$ & -0.005 & $0.565^{*}$ & $0.514^{*}$ & 1.000 & -0.077 & $0.404^{*}$ & $0.251^{*}$ & $0.274^{*}$ & $0.226^{*}$ & -0.011 \\
DD & -0.061 & -0.052 & 0.032 & $-0.113^{*}$ & -0.010 & -0.077 & 1.000 & 0.034 & 0.053 & $-0.112^{*}$ & 0.055 & 0.043 \\
PCC & $0.310^{*}$ & $-0.224^{*}$ & -0.011 & $0.349^{*}$ & $0.399^{*}$ & $0.404^{*}$ & 0.034 & 1.000 & $0.307^{*}$ & $0.242^{*}$ & $0.476^{*}$ & 0.006 \\
PL & $0.132^{*}$ & -0.011 & $0.328^{*}$ & $0.284^{*}$ & $0.232^{*}$ & $0.251^{*}$ & 0.053 & $0.307^{*}$ & 1.000 & $0.515^{*}$ & $0.301^{*}$ & $0.202^{*}$ \\
AP & 0.060 & $-0.207^{*}$ & $0.108^{*}$ & $0.310^{*}$ & $0.186^{*}$ & $0.274^{*}$ & $-0.112^{*}$ & $0.242^{*}$ & $0.515^{*}$ & 1.000 & $0.274^{*}$ & $0.158^{*}$ \\
AT-III & 0.179 & $-0.139^{*}$ & $-0.121^{*}$ & $0.323^{*}$ & $0.272^{*}$ & $0.266^{*}$ & 0.055 & $0.476^{*}$ & $0.301^{*}$ & $0.274^{*}$ & 1.000 & -0.009 \\
P & $-0.107^{*}$ & $0.095^{*}$ & $0.490^{*}$ & $0.179^{*}$ & $-0.127^{*}$ & -0.011 & 0.043 & 0.006 & $0.202^{*}$ & $0.158^{*}$ & -0.009 & 1.000 \\
\hline
\end{tabular}

P: platelets; PT: prothrombin time; PTT: partial thromboplastin time; F: fibrinogen; F-II: factor II; F-VII: factor VII; F-X: factor X; DD: D-dimer; PCC: proteic C clotting; PL: plasminogen; AP: antiplasmin; AT-III: antithrombin III; *: significant results.

median survival (7.7 months, CI: 6-9) than the others (10 months CI: 9-12, p<0.043) (fig. 1c). Also, patients with platelet counts $>289,000 \mathrm{~mm}^{3}$ survived for a shorter period (8 months, CI: 7-10) than those with lower counts (10 months, CI: 6-13, p<0.047) (fig. 1d). Multivariate analyses of survival were performed without prior dichotomization or categorization of the continuous variables. A Cox's proportional hazards regression analysis, including 293 cases and all the 32 considered variables, selected, as prognostically significant, in order of descending importance: 1) stage of
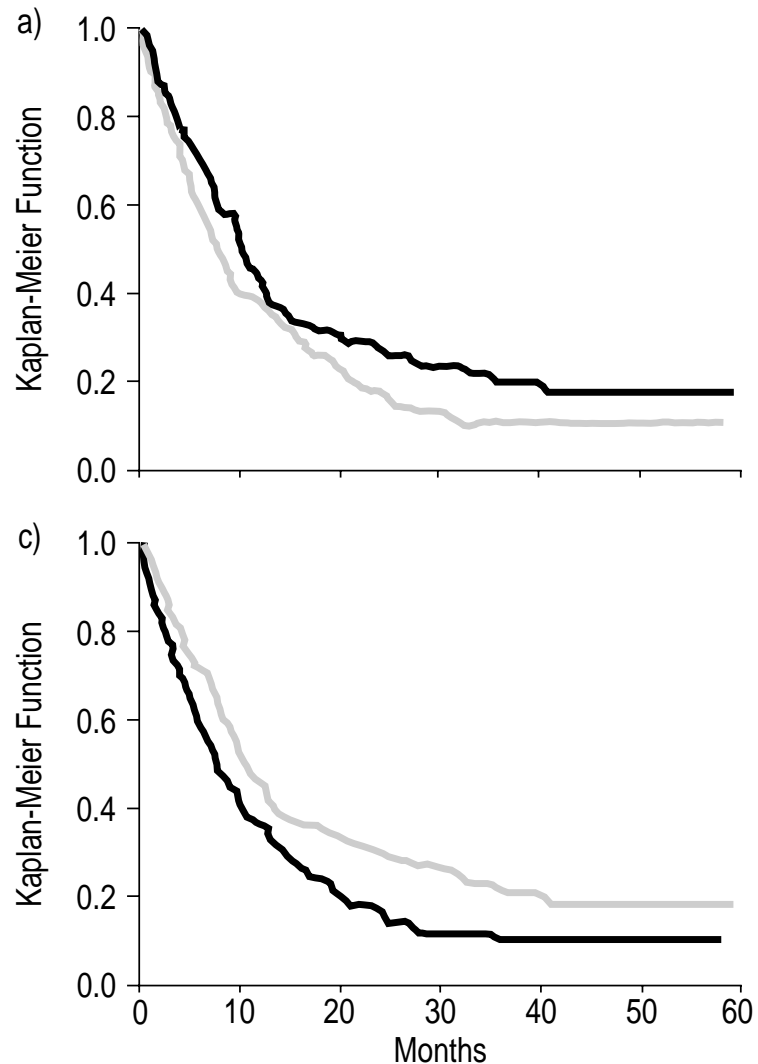

disease; 2) ECOG PS; 3) lactate dehydrogenase; 4) neutrophils; 5) tissue polypeptide antigen; and 6) creatinine (table 4).

\section{Discussion}

In lung cancer, prognostic factors are helpful in advising individuals, choosing treatments, understanding the disease and providing direction of further study. Traditionally, a number of baseline clinical

b)

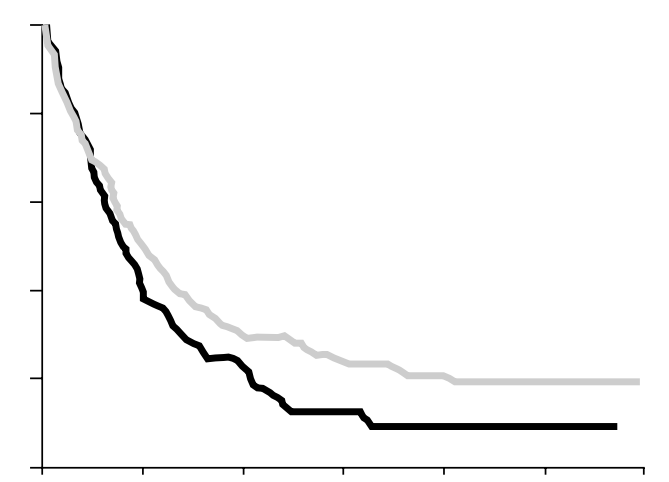

d)

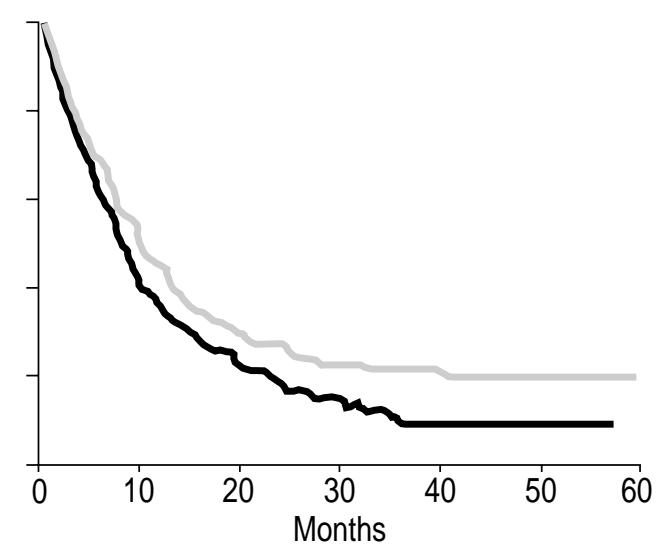

Fig. 1. - Survival probability based on a) prothrombin time ( Log rank=4.03, $\mathrm{p}=0.043)$; b) fibrinogen concentration (Log rank=4.61, $\mathrm{p}=0.031)$; c) $\mathrm{D}$-dimer concentration $(\log \operatorname{rank}=8.55, \mathrm{p}=0.003)$; d) platelet count $(\log$ rank=3.92, $\mathrm{p}=0.047)$. Dark line: values above the median; shaded line: values equal to or below the median. 
Table 4. - Factors independently influencing survival

\begin{tabular}{lcccr}
\hline Variables & Statistic & Relative Risk & $95 \%$ confidence interval & p-value \\
\hline Stage of disease & 41.5387 & 1.3090 & $1.2061-1.4207$ & 0.0000 \\
ECOG Performance Status & 15.5800 & 1.4012 & $1.1851-1.6567$ & 0.0001 \\
Lactate dehydrogenase & 11.0270 & 1.0005 & $1.0002-1.0008$ & 0.0009 \\
Neutrophils & 14.2559 & 1.0001 & $1.0000-1.0001$ & 0.0002 \\
Tissue polypeptide antigen & 9.6921 & 1.0007 & $1.0003-1.0011$ & 0.0019 \\
Creatinine & 4.3094 & 1.5312 & $1.0241-2.2894$ & 1.8271 \\
\hline
\end{tabular}

Results of a Cox's multifactorial analysis including all the 32 variables described in tables $1-2 *(* 293$ cases included) global Chi-squared $=142.066 ; \mathrm{p}=0.0000$.

characteristics such as the anatomical extent of the disease, performance status and weight loss have been used to predict the outcome in individual patients [21]. The purpose of this prospective study was to determine the possible prognostic value of 12 tests of blood coagulation (i.e., P, PT, PTT, AT-III, F, DD, F-II, FVII, F-X, PCC, PL, and AP).

The blood coagulation scheme has been divided into two pathways, "extrinsic" and "intrinsic" both of which converge in a final common pathway for the production of thrombin. Although it is operationally convenient to think of these as separate, many of their reactions suggest a fair amount of "crosstalk" between the two pathways. The term "extrinsic" implies that extra vascular substances are required to initiate blood coagulation; "intrinsic" indicates that all components required to trigger blood clotting are contained within the vascular system [22]. Approximately $90 \%$ of cancer patients with metastatic disease and half of all cancer patients have abnormal coagulation parameters [23]. Several laboratory abnormalities including prolonged and shortened PT and PTT, increased and decreased levels of F, F-V, F-VIII, F-IX, F-XI, F-XII, fibrinogen, fibrinogen/fibrin degradation products, thrombinantithrombin III complex, and thrombocytosis have been reported [23]. Thrombocytosis is found in patients with metastatic disease and has been related to extensive disease either in lung cancer or in other malignancies [24]. Recently, Pedersen et al. [24] reported increased platelet counts in $32 \%$ of 1,115 patients with primary lung cancer, and showed that thrombocytosis was prognostically significant. In the present cohort, an elevation of the platelet count above $400,000 \cdot \mathrm{m}^{-3}$ was found in 44 of the 343 assessable cases $(13 \%)$ and a poor prognosis was found for those with platelet counts above the median. Hyperfibrinogenaemia is found in the same clinical states that usually accompany thrombocytosis [22]. If the $\mathrm{F}$ level is normal, a prolonged PT signifies a deficiency of one factor of the extrinsic pathway (i.e. factor VII), as well as a deficiency of factors common to the extrinsic and intrinsic systems (i.e. factor $\mathrm{X}$, factor $\mathrm{V}$ and prothrombin) [22]. Alternatively, a prolonged PT may be due to the presence of a specific inhibitor of one of those factors, or a level of fibrin split products sufficiently high to delay fibrin formation [22]. In the presented study, a significant reduction of PT was observed in 15 cases $(4 \%$ of the sample) and an abnormally prolonged PT was strongly predictive of a bad prognosis. The smallest unique degradation product of cross-linked fibrin is fragment DD (D-dimer) which results from the proteolytic actions of plasmin on fibrin [25]. It is considered a sensitive marker of the fibrinolytic enhancement [25]. It was found that many patients $(55 \%)$ had an increased plasma level of DD, and this was associated with a poor prognosis. The partial thromboplastin time, assuming a normal fibrinogen level, screens for deficiencies of factors in the intrinsic system (XII, XI, IX, VIII) or deficiencies of factors common to the intrinsic and extrinsic system [22]. In the present study, only seven of 342 patients had abnormally prolonged PTT.

There are numerous reports indicating that lung cancer patients have an increased propensity to clotting and fibrinolytic system aberrations [2-4]. In addition, both clinical and experimental evidence supports the idea that the activation of coagulation and fibrinolysis may play an important role in the invasiveness of cancer [23]. Regarding the pathogenetic mechanism of these tumour-associated coagulopathies, many factors have been ascribed as potential causative agents. Activation of the clotting cascade may be due to tumour cells themselves, or to the stimulation of tumour associated inflammatory cells [26]. A number of studies have also shown relationships between coagulation changes and the natural history of malignancies. The hypothesis that anticoagulant therapy may prolong survival SCLC patients has been proved $[6,7]$. In the last few years, several reports have evaluated the possible prognostic significance of blood coagulation [10, 27-30]. MEEHAN et al. [27] studied 119 untreated SCLC patients and showed that higher pretreatment fibrinogen levels correlated significantly with advanced stages of disease and reduced survival. In addition, pretreatment levels were correlated significantly with the likelihood of achieving disease regression after chemotherapy. The group of SEITZ et al. [10] reported that thrombinantithrombin complex (TAT) and DD were significantly increased in metastatic disease, and that elevated TAT values at diagnosis might be prognostically significant. WoJTUKIEWICZ et al. [29] showed that survival is reduced in nonsmall cell lung cancer (NSCLC) patients having elevated value of fibrinogen and fibrin split products, platelet count and activated PTT. In patients with disseminated SCLC, these authors also reported that prolonged PTTs were associated with shortened survival. In multivariate analysis, PTT was an independent predictor of survival in limited NSCLC and in disseminated SCLC patients, while fibrin(ogen) split products were significant only in disseminated NSCLC group. TAGUCHI et al. [30] assessed the plasma level of the plasmin- $\alpha_{2}$-plasmin inhibitor complex and showed that its abnormality was a predictor of survival 
independently of stage of disease, sex, age, histological type, performance status, tumour size and presence of distant metastasis. The same group [28] measured the plasma levels of DD in 70 lung cancer patients and found that low values were significantly predictive of both good prognosis and longer survival. It has been reported, recently, that several clotting tests were predictive of prognosis, in both univariate and multivariate models [3]. Univariate tests showed that lower values of PT, higher values of F, and abnormally elevated concentrations of DD were all significantly associated with an adverse outcome. Lower values of PTT were also associated with shorter survival, but this relationship did not reach the significance level $(p=0.1)$. The multivariate models confirmed several clotting tests as significant [3].

The current study confirms that subclinical changes in the coagulation-fibrinolytic system are often present in lung cancer. The most frequent alterations concern DD, F, AT-III $(55,42$, and $28 \%$ of the cases), followed by F-VII (27\%), F-X (20\%), and F-II (16\%). Confirming our previous report, univariate analyses of survival showed that a prolonged PT and higher P, F, and DD were all associated with a poor prognosis. However, in this study, the multivariate model did not confirm the prognostic relevance of any of those coagulation factors. This may be explained by an overshadowing of their prognostic potential by the presence of other more potent recognized prognostic factors, such as ECOG performance status and TNM-stage The earlier results showing the independent prognostic significance of PT time and P [3] are, therefore, not confirmed by the present study, which was carried out on a different cohort sample and with different laboratory methods and based on a different mix of recorded variables.

Further large studies on specific subgroups of lung cancer are needed to better define the effective prognostic values of the clotting abnormalities and to design additional studies to implement the therapeutic interventions aimed to correct the activation of the clottingfibrinolytic system.

Acknowledgements. The authors thank L. Gribaudo and A. Merlo, nurses of their outpatient unit, for the invaluable help and support, and A. Cerchietti for English editing. They would also like to thank the Cuneo Lung Cancer Study Group for the technical and financial support.

\section{References}

1. Trousseau A. Phlegmasia alba dolens. Clinique Medicale de 1'Hotel-Dieu de Paris. Paris: J-B. Balliere et Fils 1865; 654-712.

2. Gabazza EC, Taguchi O, Yamakami T, Machishi M, Ibata $\mathrm{H}$, Suzuki S. Evaluating prethrombotic state in lung cancer using molecular markers. Chest 1993; 103: 196-200.

3. Buccheri G, Ferrigno D, Ginardi C, Zuliani C. Haemostatic abnormalities in lung cancer: prognostic implications. Eur J Cancer 1997; 33: 50-55.
4. Bick RL. Coagulation abnormalities in malignancy: a review. Sem Thromb Hemost 1992; 18: 353-372.

5. Pavey SJ, Hawson GAT, Marsh NA. Alterations to the fibrinolytic enzyme system in patients with nonsmall cell lung carcinoma. Blood Coagul Fibrinolysis 1999; 10: 261-267.

6. Zacharski LR, Henderson WG, Rickles FR, et al. Effect of warfarin anticoagulation on survival in carcinoma of the lung, colon, head and neck, and prostate. Final report of VA Cooperative Study \#75. Cancer 1984; 53: 2046-2052.

7. Lebeau B, Chastang C, Brechot J-M, Capron F, Dautzenberg B, Delaisements C, et al. Subcutaneous heparin treatment increases survival in small cell lung cancer. Cancer 1994; 74: 38-45.

8. Zacharski LR, Memoli VA, Costantini V, Wojtukiewicz MZ, Ornestein DL. Clotting factors in tumor tissue: implications for cancer therapy. Blood Coagul Fibrinolysis 1990; 1: 71-78.

9. Gabazza EC, Taguchi O, Yamakami T, et al. Alteration of coagulation and fibrinolysis systems after multidrug anticancer therapy for lung cancer. Eur $J$ Cancer 1994; 30A: 1276-1281.

10. Seitz R, Rappe N, Kraus M, et al. Activation of coagulation and fibrinolysis in patients with lung cancer: relation to tumour stage and prognosis. Blood Coagul Fibrinolysis 1993; 4: 249-254.

11. World Health Organization. International histological classification of tumours. Berlin, Springer Verlag, 1991.

12. Mountain CF. Revisions in the International System for Staging Lung Cancer. Chest 1997; 111: 1710-1717.

13. Zubrod CG, Scheiderman MA, Frei E, et al. Appraisal of methods for the study of chemotherapy in man: comparative therapeutic trial of nitrogen mustard and triethylene thiophosphoramide. J Chron Dis 1960; 11: 7-33.

14. Ferrigno D, Buccheri G. Is the MVP regimen less active than previously described? Results of a phase II study in advanced non-small cell lung cancer. Acta Oncol 1996; 35: 435-439.

15. Buccheri G, Ferrigno D. Vinorelbine in elderly patients with inoperable non small cell lung carcinoma. Cancer 2000; 88: 2677-2685.

16. Ferrigno D, Buccheri G, Biggi A. Serum tumour markers in lung cancer: history, biology and clinical applications. Eur Respir J 1994; 7: 186-197.

17. Siegel S. Nonparametric statistics for the behavioural sciences. New York, MacGraw Hill, 1956.

18. Kaplan EL, Meier F. Non-parametric estimation from incomplete observations. J Am Stat Assoc 1958; 58: 457-481.

19. Peto R, Pike MC, Armitage P. Design and analysis of randomized clinical trials requiring prolonged observation of each patient: II Analysis and examples. $\mathrm{Br}$ J Cancer 1977; 35: 139.

20. Cox DR. Regression models and life tables. $J R$ Stat Soc 1972; 34: 187-220.

21. Buccheri G, Ferrigno D. Prognostic factors in lung cancer: tables and comments. Eur Respir J 1994; 7 : $1350-1364$.

22. Owen CAJ, Bowie EJW, Thompson JHJ. The diagnosis of bleeding disorders. Boston: Little, Brown \& Company, 1975.

23. Gouin-Thibault I, Samama MM. Laboratory diagnosis of the thrombofilic state in cancer patients. Sem Thromb Hemost 1999; 25: 167-172.

24. Pedersen LM, Milman N. Prognostic significance of 
thrombocytosis in patients with primary lung cancer. Eur Respir J 1996; 9: 1826-1830.

25. Francis CW, Marder VJ. Mechanism of fibrinolysis. In: McGraw Hill., ed. Williams Hematology. 1995; 1252-1260.

26. Zacharski LR, Wojtukiewicz MZ. Costantini Pathways of coagulation/fibrinolysis activation in malignancy. Sem Thromb Hemost 1992; 18: 104-106.

27. Meehan KR, Zacharski LR, Moritz TE, Rickles FR. Pretreatment fibrinogen levels are associated with response to chemotherapy in patients with small cell carcinoma of the lung: Department of Veterans Affairs Cooperative Study 188. Am J Hematol 1995; 49: 143148.
28. Taguchi O, Gabazza EC, Yasui H, Kobayashi T, Yoshida M, Kobayashi H. Prognostic significance of plasma D-dimer levels in patients with lung cancer. Thorax 1997; 52: 563-565.

29. Wojtukiewicz MZ, Zacharski LR, Moritz DM, Edwards RL, Rickles FR. Prognostic significance of blood coagulation tests in carcinoma of the lung and colon. Blood Coagul Fibrinolysis 1992; 3: 429437.

30. Taguchi O, Gabazza EC, Yoshida M, Yamakami T, Kobayashi H, Shima T. High plasma level of plasminalpha2-plasmin inhibitor complex is predictor of poor prognosis in patients with lung cancer. Clin Chim Acta 1996; 244: 69-81. 